\title{
Andromaque et Bérénice: \\ les femmes raciniennes \\ sont-elles aristotéliciennes?
}

\section{Bérénice LE MARCHAND}

$\mathbf{J}$

Jean Racine, orphelin dès son plus jeune âge, reçoit une éducation influencée par les Anciens et leur austère morale, ce qui orientera sa vision du monde tragique. Andromaque et Bérénice, deux de ses chefs-d'oeuvre, représentent la tradition théâtrale à l'âge classique. Ces deux pièces, composées respectivement en 1667 et 1670, portent le nom d'une femme. Six des onze tragédies de Racine ont pour titre un prénom de femme. Andromaque, femme amoureuse de son défunt mari Hector, cède aux avances de Pyrrhus et se marie avec lui pour sauver la vie de son fils, Astyanax. Bérénice, reine de Palestine, renonce à ses amours pour Titus et Antiochus. La personnalité et la force de caractère de ces deux feinmes sont surprenantes. Mises en rapport avec la théorie d'Aristote qu' 'il ne convient pas qu'une femme soit virile ou éloquente" ( $L a$ Poétique 85), Bérénice et Andromaque font figures d'antithèse. Je me propose de développer ce contraste en analysant les facettes de la femme racinienne à l'âge classique à travers ces deux tragédies. La psychologie, l'apparence physique et les actions de ces femmes influencent l'intrigue et démontrent combien Andromaque et Bérénice sont intelligentes et fortes moralement. Cette étude, appuyée sur les définitions et influences aristotéliciennes, repose sur une mise en parallèle des deux femmes, mais aussi sur une comparaison avec les réactions et la présence masculines dans ces deux oeuvres raciniennes. 
Andromaque et Bérénice sont tout d'abord définies comme femmes énigmatiques voire mystérieuses. Andromaque de Troie et Bérénice de Palestine sont toutes deux étrangères. Leur identité est quasi-réduite à leurs origines, elles ne sont plus Andromaque et Bérénice mais la "Phrygienne" (Andromaque 572)' et celle qui n'a pas "le coeur d'une Romaine" (Bérénice 375). ${ }^{2}$ Intrigantes par leur différence, elles provoquent le malaise et le désordre dans le royaume. Hermione se révolte en pensant: "si d'une Phrygienne [Andromaque] il [Pyrrhus] devenait l'époux!" (572) et Phénice prévient Bérénice que "Rome [la] voit ... avec des yeux jaloux" (293). De plus, Bérénice, par raison d'État, ne peut se marier avec Titus puisque "l'hymen chez les Romains n'admet qu'une Romaine" (295) et par conséquence "Rome ne l'attend pas pour son impératrice" (372). Elles sont rejetées et étiquetées comme personnes suspectes et provoquant la méfiance malgré leur beauté envoûtante.

Dans ces deux tragédies, la femme est mise en avant grâce aux "charmes tout-puissants" d'Andromaque (351) et "aux beautés" (375) de Bérénice. D'elles émane la perfection physique, mais aussi l'effet sensuel qui charme les hommes. L'adjectif "belle" et le substantif "beauté" sont utilisés chacun trois fois pour qualifier Bérénice. Andromaque est une jeune mère et son charme attire plus que sa "beauté" (659). Elles deviennent objet sensuel puisque ces deux Vénus sont réduites à leurs qualités physiques lorsque Titus en parlant de Bérénice souligne que "Rome contre les rois de tout temps soulevée dédaigne une beauté" (724) et lorsqu'Oreste en évoquant Andromaque révèle "quelle joie d'enlever à l'Épire une si belle proie!" (598). Objet de désir, voire même objet de représentation faisant figures de femmes parfaites, "beauté, gloire, vertu, je trouve tout en elle" (Bérénice 544), elles ne sont qu'images séduisantes.

Également, la vertu est une de leurs qualités communes. Le mot "vertu" est utilisé quatre fois pour décrire Bérénice pleine de bonté et d'anabilité, "elle a mille vertus" (376). Andromaque est vertueuse par rapport à sa fidélité pour son défunt époux, "par cent fois le nom d'Hector est sorti de sa bouche" (650). Aristote définit les personnages d'une tragédie selon leur qualité :

... il y aura caractère si les paroles ou l'action révèlent un choix déterminé: le caractère aura de la qualité si ce choix est de qualité. Et cela est possible pour chaque genre de personnes; en effet une femme peut-être de qualité, un esclave aussi, même si l'on peut dire que l'une est un être inférieur, l'autre un être tout à fait bas. (La Poétique 85) 
Le respect d'Andromaque pour son défunt mari et la bonté d'esprit de Bérénice reflètent des personnages de qualité aristotélicienne.

Pour renforcer cette idée, rappelons que l'humorisme qui fait reposer tous les états du corps et de l'âme sur les humeurs persiste encore au XVIIème siècle. Cette théorie basée sur les quatre humeurs (sang, phlegme, bile jaune et bile noire) et les qualités physiques qui les accompagnent (chaud, froid, sec et humide) définissaient les personnalités et les différences mêmes entre les hommes et les femmes. Cette doctrine, avancée par Aristote et développée par Galen, souligne que "la femelle est plus imparfaite que le masle, pour une et principale raison, à savoir pource qu'elle est plus froide" (833). En reprenant cette vision aristotélicienne, on note que les fluides d'Andromaque et Bérénice ne sont pas en harmonie. Leurs réactions passionnées et extrêmes démontrent le déséquilibre de leurs humeurs. Ainsi, on note par exemple la série de questions de Bérénice à l'acte II scène 5 lorsqu'elle apprend que Titus la rejette, "Qu'ai-je fait? Que veut-il? Que dit ce silence?" (627) et "n'ai-je rien dit qui lui puisse déplaire?" (636) qui indique son émoi et la difficulté qu'elle a à contrôler son trouble. Également, on relève la tristesse continue d'Andromaque, "ses pleurs éternels" (304) et son amour passionné pour son défunt mari. Andromaque est affectée par cette perte et ne semble pas pouvoir se maîtriser. Ces réactions s'accordent avec la pensée d'Aristote; une fenme est, "more compassionate than man, more easily moved to tears, ... . more jealous, more querulous, more apt to scold and to strike. The female is also more despondent and despairing than the male, more shameless and more given to falsehood, more easily deceived and of more retentive memory" (History of Animals 608b. 8-15). Nancy Tuana dans son article, "Aristotle and the Politics of Reproduction," souligne qu'"Aristotle simply inferred from the conjunctions of these beliefs that a female must be the result of some lack of generative heat or some adversity. On this account, woman is a misbegotten man, resulting from some defect in the heat of the generative process" (200). Néanmoins, Bérénice se soucie d'avoir "avec trop de chaleur rabaissé [les] présents [de Titus] ou blâmé sa douleur" (Bérénice 637). L'utilisation du mot "chaleur" est directement liée à la tradition de l'humorisme qu'Aristote prône lorsqu'il affirme que "a defect in heat resulted in woman being born a "mutilated male"" (Generation of Animals 775a. 14-21). Ici, Bérénice manifeste trop de chaleur et deviendrait même quasi-homme. De même, le "désordre extrême" dont Phénice parle en se référant à Bérénice, "mais voulez-vous paraître en ce désordre extrême?" (Bérénice 967), appartient à ce lexique de déséquilibre entre les fluides. 
De même, elles sont toutes les deux amoureuses. La qualité aristotélicienne est renforcée lorsque Bérénice ne peut concevoir sa vie sans Titus, "un soupir, un regard, un mot de votre bouche, voilà l'ambition d'un coeur comme le mien" (577) et lorsqu'Andromaque ne vit que grâce à son souvenir d'Hector, "as-tu pensé qu'Andromaque infidèle put trahir un époux ... ?" (1077). Leurs amours sont quelque peu différents puisque celui de Bérénice pour Titus est passionné et reflète la jeunesse et la vigueur d'une jeune femme en plein bonheur. Descotes remarque qu'Andromaque, elle, exprime "une fidélité à un devoir, l'attachement à la patrie perdue ... sa volonté de conserver le dernier témoignage d'un bonheur disparu" (5).

Andromaque et Bérénice portent en elles le potentiel de tuer. La confidente d'Andromaque remarque que "trop de vertu pourrait [la] rendre criminelle" (982) et Barthes dans son ouvrage Sur Racine écrit "puissante, Bérénice tuerait" (95). Ces deux femmes deviennent de vraies guerrières prêtes à défendre leurs intêrets par le crime. La veuve d'Hector sacrifierait son fils pour respecter son défunt époux tandis que la promise de Titus s'écrit "moi je vivrai Phénice" (645) et "tu verras de quel prix ton coeur est à mes yeux" (662). Déterminées, Andromaque et Bérénice sont femmes raciniennes dans le sens où elles vont à l'encontre de la théorie d'Aristote qu'"un caractère peut être viril, mais il ne convient pas qu'une femme soit virile ou éloquente" (Poétique 85). Elles deviennent quasi-hommes lorsqu'Andromaque déclare, "as-tu pensé qu'Andromaque infidèle put trahir un époux qui croit revivre en elle" (107-08). Elle vit pour Hector ou plutôt Hector vit en elle. Elle puise sa force dans le souvenir d'Hector lui permettant ainsi d'être intouchable, "c'est votre époux, madame, qui produit ce miracle en votre âme" (1049-50) et "il vous rend souverraine" (1057). En s'écriant, "allons rejoindre mon époux" (924), Andromaque évoque le tombeau de son défunt époux. Néanmoins, Hector étant une partie d'elle-même, il n'est pas difficile de conjecturer qu'elle souhaite la mort pour être enfin réunie à jamais avec son bien-aimé. Ce procédé métonymique met en relief le lien étroit unissant Andromaque et Hector mais également l'aspect transcendant d'Andromaque: "s'il me perd, je prétends qu'il me retrouve en toi" (1024). Parallèlement, Bérénice s'associe entièrement à Titus, et " $n$ 'aime en lui que lui-même" (160) et "son coeur n'a jamais demandé que le mien" (530). Son amour passionné constitue sa force physique et morale. Bérénice déclare même, "moi qui mourrais le jour qu'on voudrait m'interdire de vous. .." (615). Elle fait partie intégrante de Titus et si elle était séparée de lui cela provoquerait sa mort, "Titus m'aime. Titus ne veut point que je meure" (911). 
Également, ces femmes se déclarent toutes les deux "éperdues": "peins-toi dans ces horreurs Andromaque éperdue" (1005) et "vous voyez devant vous une reine éperdue" (Bérénice 872). L'adjectif "éperdu" définit une personne vivement émue, troublée par une violente émotion ou égarée (Petit Larousse Illustré 373). Andromaque, face au dilemme de choisir entre la vie ou la mort de son fils, se décrit comme "captive, toujours triste, importune à [elle]-même" (301) et ses pleurs semblent incessants. Bérénice, elle, ne peut concevoir sa vie sans Titus: "nous séparer? Qui? Moi? Titus de Bérénice?" (895), et elle essaie de comprendre ce rejet soudain: "ce piège n'est tendu que pour nous désunir" (910).

De plus, elles se forgent une facette de femmes victimes où la tristesse et le malheur semblent constituer leurs vies. Les mots liés au champ lexical de la tristesse et du mallheur sont utilisés seize fois pour se référer à Andromaque et trente-cinq fois pour décrire Bérénice. Par exemple, on note pour Andromaque "pleurs éternels," "mes larmes," "toujours triste" (Andromaque 301, 304, 362), et pour Bérénice "ses pleurs," "ses malheurs" et "la triste Bérénice" (Bérénice 745, 802, 972). Ce contraste numéral est directement lié à leurs différentes approches manipulatrices. Andromaque, plus introvertie, prépare sa défense intérieurement. Bérénice, elle, choisit les mots comme armes de combat. Toutes les deux se créent un univers pathétique où la mort semble leur compagne les guidant aveuglément vers le trépas. Le substantif "mort" est utilisé deux fois pour Andromaque et cinq fois pour Bérénice; ainsi on remarque: "elle en mourra" (Andromaque 698) et "la Reine veut mourir" (Bérénice 1200). L'utilisation des adjectifs possessifs renforce la douleur qui émane de leur personnalité: "mes pleurs ... mes gémissements ... mes pleurs ... ma perte ... ma mort" (Bérénice 974-76) ou encore "mes pleurs" (Andromaque 338) et "mes larmes" (Andromaque 362). Cet égocentrisme permet de renforcer leur aspect pathéthique et leur affliction.

Néanmoins, se transformant en femmes caméléons, elles projettent une fausse image de leur caractère. Cette fragilité et cette faiblesse ne sont qu'une illusion. On apprend dès la première scène qu'“Andromaque trompa l'ingénieux Ulysse" (74) en faisant tuer un autre enfant et non pas son fils Astyanax. Dès lors on sait qu'Andromaque a la capacité d'agir et de se battre. Par conséquent, l'élaboration de son plan pour piéger Pyrrhus ne devrait pas surprendre:

Je vais donc, puisqu'il faut que je me sacrifie

Assurer à Pyrrhus le reste de ma vie, Je vais, en recevant sa foi sur les autels, L'engager à mon fils par des liens immortels. 
Mais aussitôt ma main, à moi seule funeste,

D'une infidèle vie abrégera le reste,

Et, sauvant ma vertu, rendra ce que je dois

À Pyrrhus, à mon fils, à mon époux, à moi.

(Andromaque 1089-96)

En acceptant le mariage, Andromaque trompe Pyrrhus. Celui-ci ne se doute pas de "l'innocent stratagème" (1097) et décide d"épouser une Troyenne" (1281) même si "Andromaque [lui] arrache un coeur qu'elle déteste" (1298). Certes, Pyrrhus croit avoir dominé sa captive, mais le spectateur se rend bien compte du pouvoir d'Andromaque à ce moment précis de l'intrigue de la pièce. C'est Andromaque qui désormais mène le jeu dramatique.

Caméléon, Bérénice le devient également lorsque, comme nous l'explique Karl Vossler, "goaded on by pain; she seizes the bridle of events, wins back her power over Titus and causes him to waver in his resolve" (69). Bérénice s'écrie: "Je n'écoute plus rien; et pour jamais, adieu" (1103) et "n'êtes-vous pas content? Je ne veux plus vous voir" (1304). Désormais, c'est Bérénice qui contrôle la situation. Notamment, à la scène dernière, Bérénice domine en toute puissance. Racine, d'habitude avare de didascalies, précise que Bérénice se lève (Bérénice 428). Cette position debout renforce sa puissance et sa domination sur Titus et Antiochus. Maîtresse du jeu, elle se permet d'ordonner "arrêtez, arrêtez" (1469) et "ne suivez point mes pas" (1505). Comme le note si justement Barthes, "Bérénice n'est donc pas une tragédie du sacrifice, mais de l'histoire d'une répudiation que Titus n'ose pas assumer" (98). Pour reprendre la citation d'Aristote, "il ne convient pas qu'une femme soit virile ou éloquente" (La Poétique 85), on note que Bérénice et Andromaque font figures d'antithèse puisqu'elles prennent des initiatives qui changent le cours de la pièce. Elles dominent la virilité de Titus et de Pyrrhus mettant ainsi en valeur leur intelligence et leur force morale.

Maurice Descotes déclare dans son ouvrage Les grand rôles $d u$ théâtre de Jean Racine, qu'"Andromaque n'évolue pas" (3). C'est à mon avis une déclaration très simpliste. Comme l'indique également Darcos, on sait que :

. . la dramaturgie tragique repose sur un principe simple: une situation inextricable et fatale au sein de laquelle des passions contraires se heurtent. L'issue [est] connue d'avance, toute l'attention se porte sur la progression logique de l'action, réduite à quelques péripéties ou coups de théâtre qui réaniment 
régulièrement l'illusion des personnages sur leur propre destin. (269)

Certes, Andromaque reste fidèle à Hector du début jusqu'à la fin tout en ayant une vie intérieure, introvertie et recroquevillée sur son amour passé qui l'a comblée. En outre, cette apparence figée n'est qu'ingénieusement prétendue. Cette réflexion calme et intérieure qui caractérise la personnalité d'Andromaque ne signifie pas qu'elle n'évolue pas. Au contraire, comme le note Castex, "Andromaque est la Parque de la pièce. Elle tire les ficelles du jeu dramatique" (292). Ses décisions contrôlent l'évolution de la pièce, elle a plein pouvoir sur Pyrrhus dans le sens où de son choix dépend le futur de ce dernier, "je meurs si je vous perds, mais je meurs sij'attends" (Andromaque 972). Il en est de même pour Bérénice qui après avoir déclaré, "je m'agite, je cours, languissante, abattue, la force m'abandonne, et le repos me tue" (955-56), décide d'affronter Titus "il est donc vrai que Titus m'abandonne?" (1043). Dès lors, ce n'est plus la Bérénice inquiète, confiant ses alarmes à Antiochus (151) ou les larmes aux yeux, mais elle devient la maîtresse du jeu dramatique mis en place par ses soins.

Par ailleurs, femmes manipulatrices, Andromaque et Bérénice, derrière leur simulacre de femmes victimes, se jouent de leurs prétendants. Toutes deux étayent leur rôle sur le registre de la perte. Andromaque, en affirmant, "non, non, j'ai beau pleurer, sa mort est résolue" (897), essaie de faire fléchir Pyrrhus. Bérénice, elle, menace de se tuer, "si devant que mourir, la triste Bérénice. .." (1188). L'on craint sa perte, "la reine veut mourir. Allons il faut la suivre" (1200) et sa menace suicidaire fait fléchir Titus qui s'alarme, "courons à son secours" (1201) et "je ne souffrirai point que Bérénice expire" (1215). Pour renforcer le pathétique de la situation, Racine trouve bon d'ajouter une didascalie au texte, "se laisse tomber sur un siège" (Bérénice 424) permettant ainsi de saisir toute la mise en scène de la faiblesse et de l'effondrement de Bérénice.

Toutefois, leurs tactiques de domination diffèrent. Andromaque parle peu puisqu'elle dit 226 vers sur les 1648 de la pièce. Andromaque incarne presque un esprit qui règne sur toutes les scènes même si elle n'est pas présente physiquement. Même présente, Andromaque donne l'image d'une femme stoïque et soumise,

Andromaque, au travers de mille cris de joie,

Porte jusqu'aux autels le souvenir de Troie;

Incapable toujours d'aimer et de haïr,

Sans joie et sans murmure elle semble obéir.

(Andromaque 1437-40) 
Les personnages d'Oreste et d'Hermione ne vivent que grâce aux actions d'Andromaque. En effet, même si la veuve d'Hector n'apparaît pas fréquemment, son esprit omniprésent contrôle tout et ces deux personnages n'ont de substance que par rapport à ses décisions. Contrairement à Andromaque, Bérénice est loquace. Celle-ci dit 386 vers sur les 1506 vers de la pièce. Cette verbalité émotionnelle se lit même sur le visage de Bérénice:

Mais voulez-vous paraître en ce désordre extrême?

Remettez-vous, Madame, et rentrez en vous-même.

Laissez-moi relever ces voiles détachés,

Et ces cheveux épars dont vos yeux sont cachés.

Souffrez que de vos pleurs je répare l'ouvrage.

(Bérénice 967-71)

Femmes aphrodisiaques, Bérénice et Andromaque deviennent femmes fatales. Leurs charmes ne les enferment pas dans la fatalité d'être à jamais des objets sensuels. Au contraire, leur beauté leur donne du pouvoir, telle Andromaque dont "la beauté . . rassure" (659) et telle Bérénice dont la beauté pourrait faire fléchir le Royaume, "Rome lui serat-elle indulgente ou sévère? ... une si belle Reine" (368-70). Leurs attraits féminins deviennent leurs atouts. Andromaque note que le "si grand coeur [de Pyrrhus] montre tant de faiblesse" (298); Pyrrhus lui-même déclare, "je trouvais du plaisir à me perdre pour elle" (642) et "je meurs si je vous perds" (972). Bérénice provoque également la faiblesse de Titus, "j'ai vu couler des pleurs qu'il voulait retenir" (965), "vous êtes empereur, Seigneur, et vous pleurez!" (1154) et "oui, Madame, il est vrai, je pleure, je soupire, je frémis" (155-56). Elles ont un tel pouvoir sur eux qu'elles se font même aimer au plus profond de leur douleur, "dans mon désespoir trouvez-vous tant de charmes?" (Bérénice 1347) et "quels charmes ont pour vous des yeux infortunés qu'à des pleurs éternels vous avez condamnés?" (Andromaque 303). Ces antithèses démontrent combien elles dominent; ce sont elles qui imposent les règles et qui jettent les dés de l'avenir.

Andromaque et Bérénice sont à l'apogée de leur domination lorsqu'elles sont toutes les deux clairement divinisées. En effet, Andromaque devient reine d'Épire ayant toute puissance, "aux ordres d'Andromaque ici tout est soumis" (1587). Bérénice, elle, en déclarant, "servons tous les trois d'exemple à l'univers/ De l'amour la plus tendre, et la plus malheureuse, dont il puisse garder l'histoire douloureuse" (150204), propose que leur histoire devienne un modèle pour le monde entier. Andromaque et Bérénice incarnent la femme racinienne détenant pouvoir 
et raison. Andromaque et Bérénice représentent la tragédie du refus où le compromis est impossible; néanmoins, comme le rappelle Vossler, d'elles émane "a growth of ethical personality" (69).

Mort à l'aube du XVIIIème siècle, Racine marque la littérature française par sa rigueur et sa maîtrise des règles de la tragédie. Il n'y a ni machineries compliquées, ni costumes chamarrés ou décors luxueux, mais un théâtre sobre et dénudé. Vossler remarque que "the power of Racine's dramatic words comes from their genuineness and simplicity. The speech of his characters is so transparent and so objective that it attains the maximum affectiveness with sparing intonation, and has no need of any magic deceit" (67). Dans toute tragédie, l'amour est impossible. Par raison d'État, Bérénice ne peut épouser Titus et Andromaque, fidèle à son défunt époux, ne connaitra plus l'amour. Ces deux femmes ont une noblesse morale touchante. Femmes gracieuses et de "qualité" (La Poétique 85), elles représentent dans ce sens la femme aristotélicienne. Néanmoins, femmes manipulatrices, caméléonnes et dominatrices elles deviennent femmes raciniennes. Jean Racine, suivant sa propre devise "la principale règle est de plaire et de toucher. Toutes les autres ne sont faites que pour parvenir à cette première" (Préface Bérénice 369), réussit à créer deux chefsd'oeuvre dont émanent deux femmes intelligentes et moralement fortes.

Vanderbilt University

\section{Notes}

1. Toutes les prochaines citations tirées de la pièce Andromaque indiqueront les vers et non les pages dans l'édition de Jean Racine, Andromaque, ed. Bernard Lalande (Genève: Droz, 1977).

2. Toutes les prochaines citations tirées de la pièce Bérénice indiqueront les vers et non les pages dans l'édition de Jean Racine, Bérénice, ed. Jean Rohou (Paris: Librairie Générale Française, 1988).

\section{Ouvrages Cités}

Aristote. La Poétique. Trans. Roselyne Dupont-Roc et Jean Callot. Paris: Éditions du Seuil, 1980.

—. Generation of Animals. Trans. Peck. Cambridge: Harvard UP, 1943.

—. Historia Animalium. Trans. Peck. Cambridge: Harvard UP, 1991.

Barthes, Roland. Sur Racine. Paris: Éditions du Seuil, 1963. 
Castex, Jean-Pierre. Histoire de la littérature française. Paris: Hachette, 1974.

Darcos, Xavier. Le XVIlème siècle en littérature. Paris: Hachette, 1987.

Descotes, Maurice. Les Grands rôles du théâtre de Jean Racine. Paris: PUF, 1957.

Galen. Selected Works. Trans. Singer. Oxford: Oxford UP, 1997.

Racine, Jean. Andromaque. Ed. Bernard Lalande. Genève: Droz, 1977.

-. Bérénice, ed. Jean Rohou. Paris: Librairie Générale Française, 1988.

Tuana, Nancy. Engendering Origins: critical feminist readings in Plato and Aristotle, "Aristotle and the Politics of Reproduction." Ed. BatAmi Bar On. New York: State U of New York P, 1994.

Vossler, Karl. Jean Racine. Trans. Isabel and Florence McHugh. New York: Frederick Ungar, 1926.

Petit Larousse Illustré. 